\title{
Understanding Influences on Decision Making Combining Cultural Aspects with Role Profiles to Evaluate How Decisions are Influenced
}

\author{
Guy Schmidt, Michael Henshaw, and Carys Siemieniuch
}

\begin{abstract}
A key issue for today's decision support tools is the increasing complexity of the environment in which industries and their supply chains operate. These entities can be classified as Enterprise Systems and Systems of Systems. The aim of this paper is to describe the development of an integrated modelling approach that could deliver greater understanding of the influences associated with the decision making process at the Enterprise System level by taking into account cultural aspects and role profiles of key stakeholders.
\end{abstract}

Index Terms - Complexity, culture, decision aiding tools, enterprise systems, influence, modelling, stakeholders.

\section{INTRODUCTION}

The INCOSE/IEEE definition of a system is: a combination of interacting elements organized to achieve one or more stated purposes [1]. The problem area today is the complexity that is building up in the development and operation of System of Systems (SoS) as defined by Jamshidi [2], one particular example of which is the Air Transport System (ATS).

A SoS is a composition; it consists of constituents that are themselves systems which possess both operational and managerial independence and can be geographically distributed [3]. These complex SoS can exhibit evolutionary behavior, leading to emergent behavior causing greater uncertainty of potential outcomes both positive and negative.

The research described in this paper, sponsored by an Innovate UK funded project, Agile Wing Integration (AWI), aims to develop an integrated approach to modeling the key stakeholders who have influence over decisions to purchase new aircraft, enabling Original Equipment Manufacturers (OEMs) to better predict customer decision outcomes.

\section{A. Problem Identification}

The development of civilian aircraft today is reaching a plateau point where the investment is delivering reduced return on investment and performance benefit. The research reported here has developed from the need within the aviation/aerospace industry to examine assessment mechanisms and is working towards the next big innovation. The aim of the AWI project is to identify areas to deliver a step jump in performance.

Manuscript received March 17, 2016; revised January 10, 2017.

Guy Schmidt, Michael Henshaw, and Carys Siemieniuch are with School of Electronic, Electrical and Systems Engineering, Loughborough University Loughborough, Leicestershire, UK, LE11 3TU (e-mail: G.J.Schmidt@lboro.ac.uk,

C.E.Siemieniuch@1boro.ac.uk).
The AWI project is a $£ 16$ million Innovate UK funded project which brings together expertise from a number of partners including Airbus, Airbus Group Innovations, Bristol University, Cranfield University, Marshall Aerospace and Loughborough University [4]. The research reported in this paper is based on work being carried out within a single work package aimed at identifying and understanding the future value drivers within the industry and to simulate and test the possible impact of introducing a disruptive innovation into the global ATS.

\section{B. Research Aim}

The aim of this research is to develop a method by which the stakeholders' interactions and influences within a SoS can be modelled; with the further objective of understanding how stakeholders' cultural attributes affect and influence decision making processes and outcomes. By understanding key activities undertaken by stakeholders within the ATS, the roles, interactions and key decision points can be established and then modelled to understand them better.

\section{Research Approach}

This research follows a mixed methods methodology. The use of both qualitative and quantitative methods enables provision of a more comprehensive research outcome. The qualitative methods provide in-depth and contextualized insights whereas the quantitative methods provide efficient, though less in-depth, predictive outcomes.

The research has iteratively analyzed appropriate literature, case studies and interviews. The building of models and frameworks and the application of these to set scenarios within an established use case(s) is currently ongoing. Fig. 1 provides a high level overview of the modelling types that have been assessed throughout the research and the aspects that are being considered.

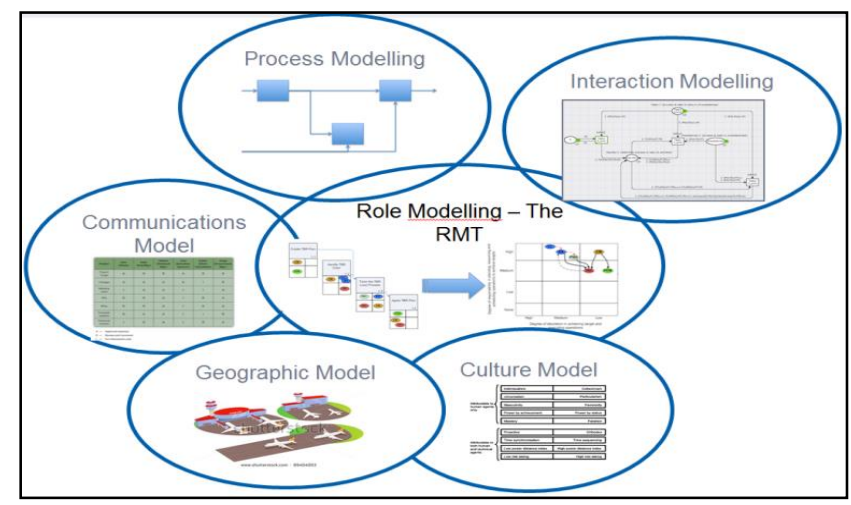

Fig. 1. Overview of modelling methods in focus. 


\section{RELATED WORK}

A number of architecture frameworks have been developed such as MoDAF [5] and DoDAF [6], to provide a framework for mapping out the architecture of Systems/SoS. MODAF provides a coherent set of rules and templates, known as 'views', that, when populated, provide a textual and graphical visualisation of the business area being investigated.

Each view offers a different perspective on the business to support different stakeholder interests.

The views are divided into 7 categories [5]:

1) Strategic views ( $\mathrm{StVs})$ define the desired business outcome, and what capabilities are required to achieve it

2) Operational views (OVs) define (in abstract rather than physical terms) the processes, information and entities needed to fulfil the capability requirements

3) Service oriented views (SOVs) describe the services, (i.e. units of work supplied by providers to consumers), required to support the processes described in the operational views

4) Systems views (SVs) describe the physical implementation of the operational and service orientated views and, thereby, define the solution

5) Acquisition views (AcVs) describe the dependencies and timelines of the projects that will deliver the solution

6) Technical views (TVs) define the standards that are to be applied to the solution

7) All views (AVs) provide a description and glossary of the contents of the architecture

In addition to these viewpoints which are static source views, work has been carried out in modelling SoS architectures and patterns to try and determine the feasibility of dynamic architectures; this work has been carried by projects such as DANSE (Designing for Adaptability and evolutioN in SoS Engineering) [7] and COMPASS (Comprehensive Modelling for Advanced SoS) [8].

Fig. 2 illustrates the methodology used within the project to form the Model Based Systems Engineering (MBSE) approach. One of the key aspects of this approach is the heavily iterative nature of the method which is required to track the ever-changing environment that SoS (such as the ATS) operate in. Due to a number of factors such as constituent systems interdependency and the emergent behavior which can occur, an iterative approach is vital as it allows adoption and refinement of the architecture and management of the SoS.

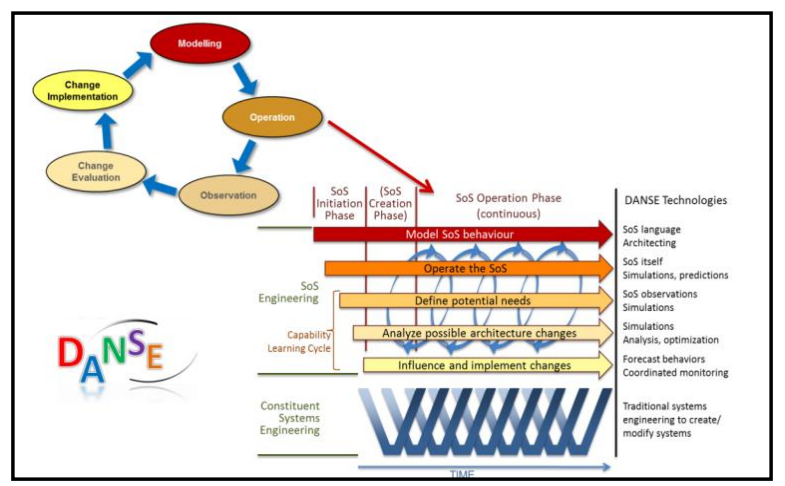

Fig. 2. DANSE MBSE methodology for SoS [9].
The emergent properties of evolutionary SoS/Enterprise Systems lead to greater uncertainty of potential outcomes both positive and negative. Within this research, the ATS is represented as a SoS with constituent systems operating within it to achieve a SoS capability. Fig. 3 shows a simplified relationship between the required capability of a SoS, its requirements and the constituent systems that comprise it.

It can be seen from Fig. 3 that emergent behavior within the constituent systems has the potential to affect the overall capability of the SoS. Being able to understand the interaction between the constituent systems and the decision making processes within the SoS would allow more efficient and effective management.

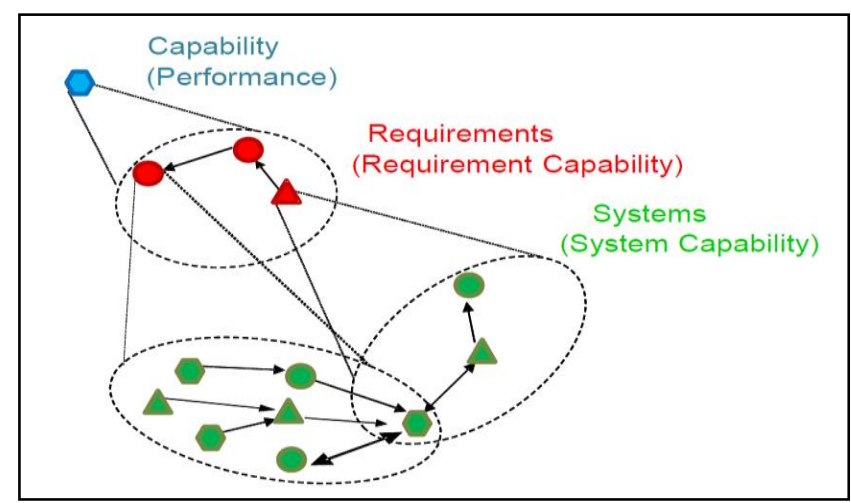

Fig. 3. Hierarchical relationship between capability, requirements and systems [10]

A critical element of an Enterprise SoS is that of the stakeholders within them and their interactions; stakeholders include the key decision makers in SoS and determine the inputs and outputs of the Systems/SoS. A number of techniques have been used to understand the interactions, influences and allocation of function between stakeholders such as social network theory [11], network modelling and Role Matrix Technique (RMT) [12] to name a few.

\section{CURRENT WORK}

One aspect of the research aims to combine cultural aspects with role profiles to evaluate how the decisions within a process are influenced in different environments. Building upon the current modelling and framework methodologies developed already, the research has investigated the possibility of combining network/architecture modelling theory with RMT properties to evaluate stakeholder viewpoints and interactions at the SoS/Enterprise System level.

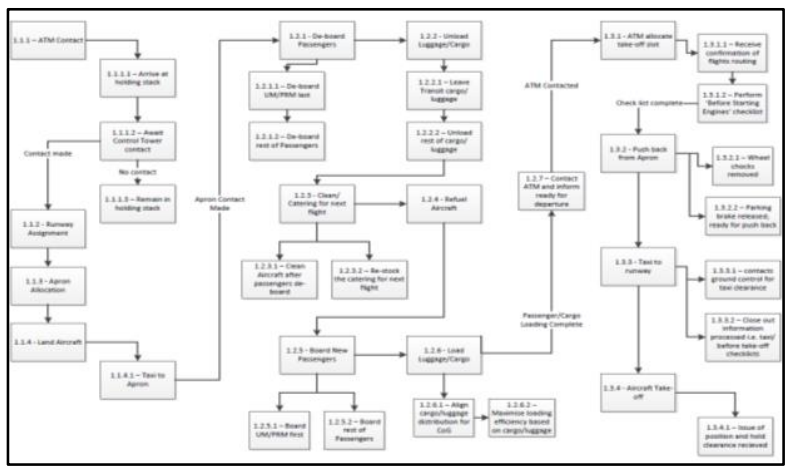

Fig. 4. Aircraft turnaround FPD. 
Once the process has been formalized the roles within the process can be defined and the key interaction points between different roles identified. To be able to identify the complexity within the role interactions an RMT was performed on one of the scenarios.

The RMT [12] incorporates some of the influences from the power versus interest grid method [14] but is able to show interactions and discern between the types of interaction. Fig. 5 and Fig. 6 illustrates the application of the RMT to the aircraft landing phase of the turnaround FPD; where roles are allocated to functions in one of four categories: controlling, providing constraining advice, providing discretionary advice and executing roles.

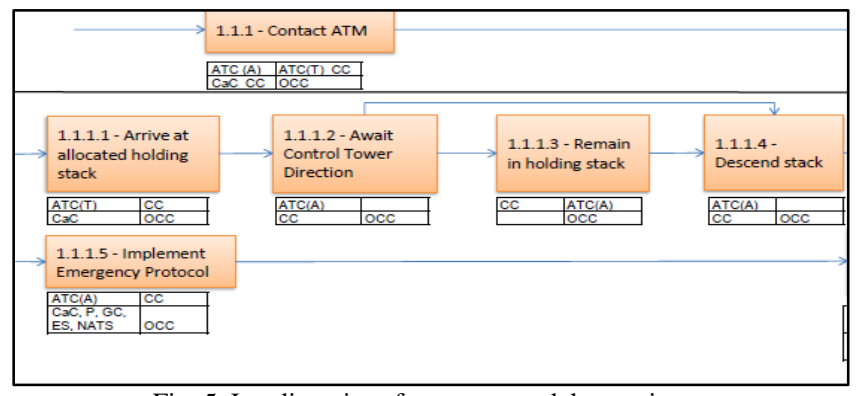

Fig. 5. Landing aircraft process model - section a.

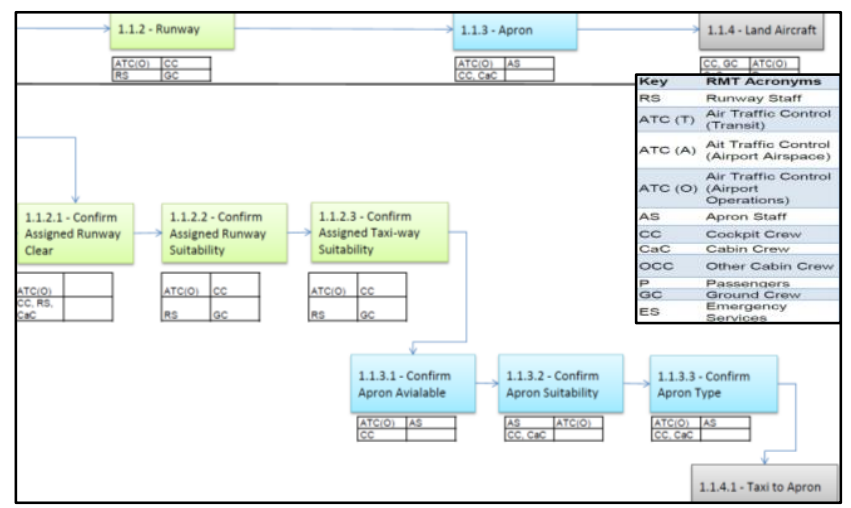

Fig. 6. Landing aircraft process model - section $b$.

The final step within the method is to transfer the roles established within the first stage to a role matrix. The role matrix aims to "create an organizational structure for a given set of roles and allows relationships between these roles for a given process to be defined' [12]. Fig. 7 is the final role matrix after aggregating up the levels of abstraction for the landing phase of the aircraft turnaround process; the key inset defines the interaction between the stakeholders.

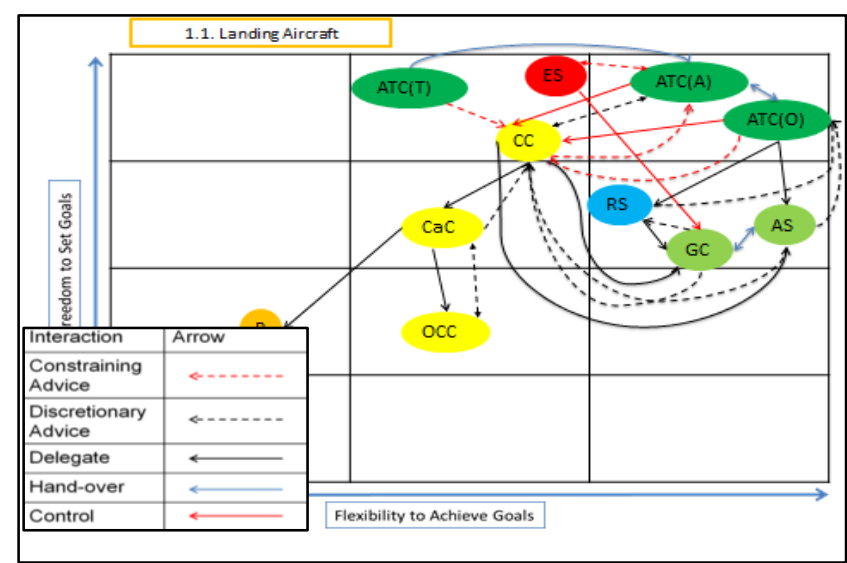

Fig. 7. Landing aircraft RMT.
The use of Coloured Petri-Nets (CPN) is also being assessed with possible integration with Bayesian Network modelling to identify the key decision points within a process and model the probability of an outcome, taking into account influences associated with the decision point and decision making agent.

The case study represents a technological disturbance, e.g. new wing design, introduced into the ATS and the impact upon the stakeholders will be evaluated and assessed to validate the modeling approach used.

'A CPN model is an executable representation of a system that can be analyzed through simulation.' [15]. The technique provides a graphical notation for modelling systems and performing analysis. CPN's have already been used to model the turnaround process within the work done by the INTERACTION project [16]. Fig. 8 shows the application of CPN modelling to the aircraft turnaround procedure.

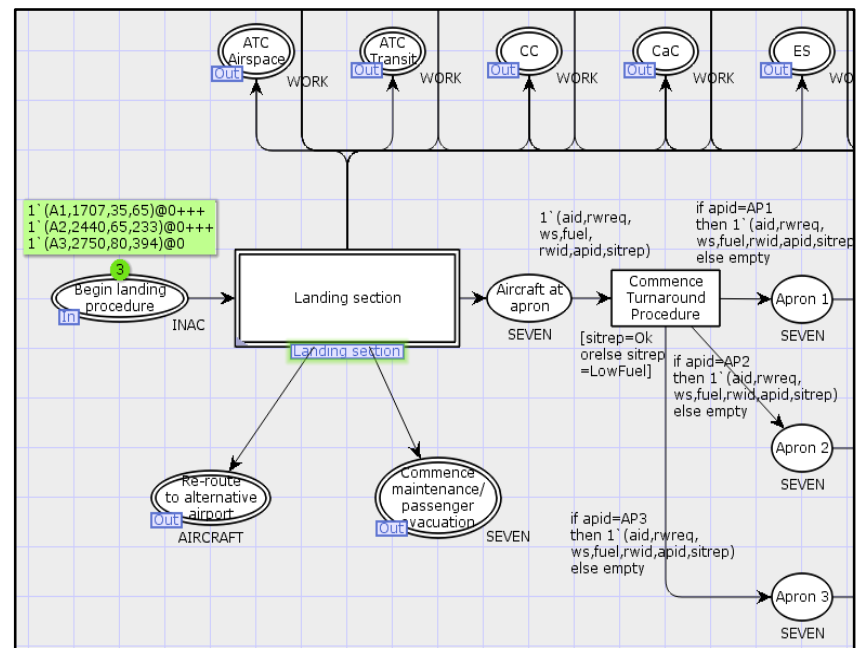

Fig. 8. Section of the CPN model of aircraft turnaround process.

CPN's show potential in being able to model stakeholder processes and interactions within the ATS and be able to incorporate multiple attributes per stakeholder. A key use of CPN modelling within the research is to identify the key decision points within the formal process developed. Fig. 9 is an example of a decision point identified within the aircraft turnaround procedure modelled using CPN.

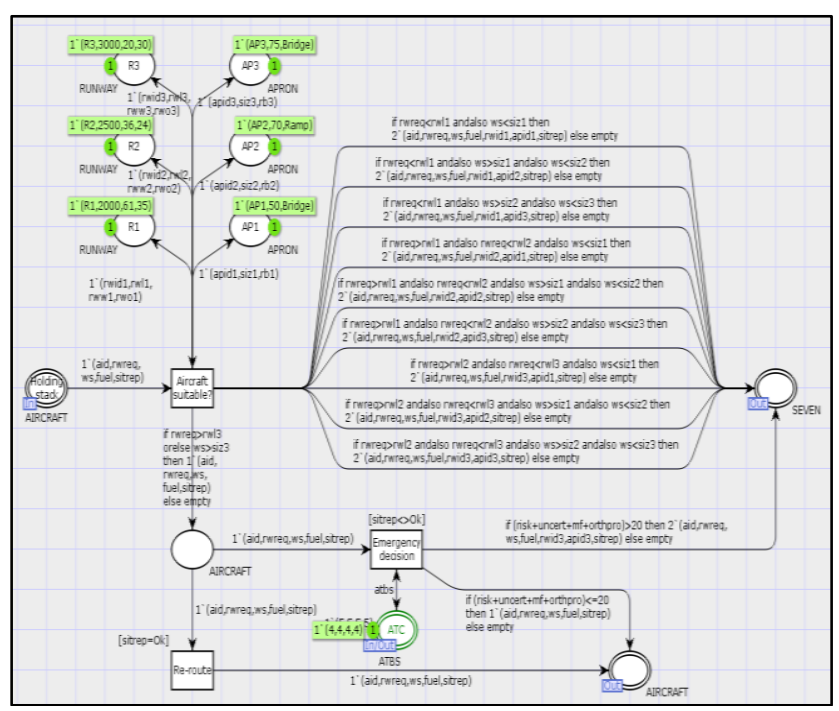

Fig. 9. Decision point - is the aircraft suitable for the airport? 
Once the key decision points have been identified, node sensitivity analysis can be carried out through the use of Bayesian Network (BN) modelling. Due to the soft nature of cultural aspects the $\mathrm{BN}$ modelling will permit the probability modelling of each of the key decision points within the process. These will provide the likelihood of a decision outcome based upon the current environment, situation and goals of the stakeholders involved.

Defining the attributes of the stakeholders within the System of Interest (SoI), which are socio-technical, is a key factor in being able to model their influence upon each other and the outcome of the key decision points because it delivers an understanding of stakeholders' goals and purpose within the ATS. Fig. 10 outlines the current modelling architecture that is still under development. The next key development is the numeration and application of cultural attributes.

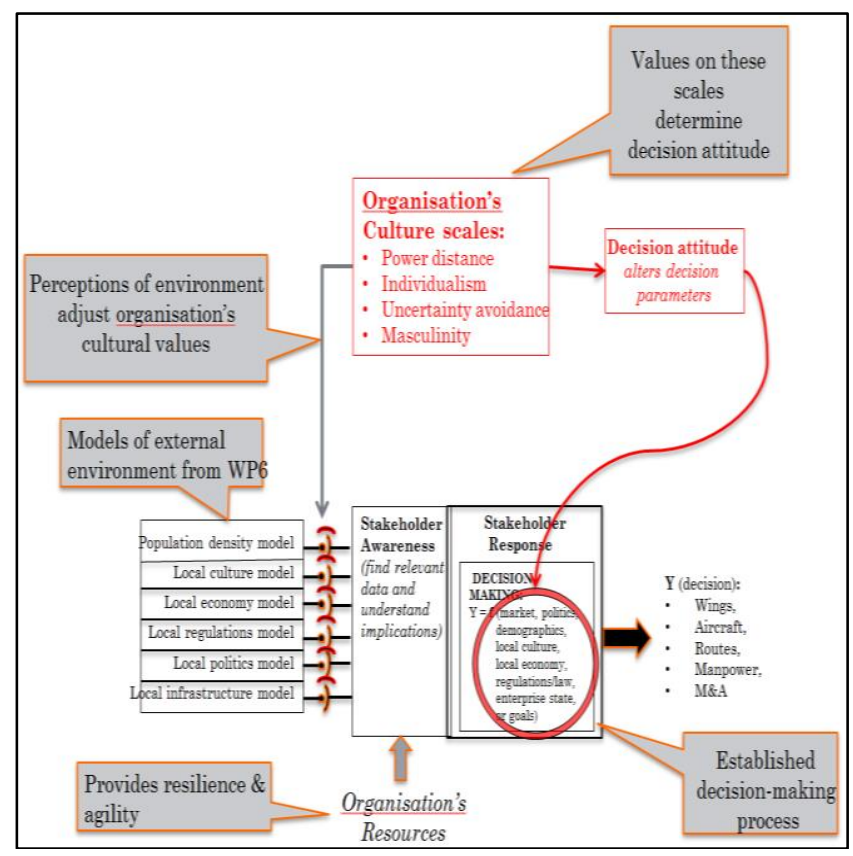

Fig. 10. Stakeholder decision influence model architecture.

\section{SUMMARY}

The key focus of the research is the combination of role profiles for stakeholders and the cultural aspects that are inherent within them. The research completed thus far has developed a methodology for the development of the role profiles and architecture for a modelling approach that could be used. The factor of cultural attributes is the next stage of the research. The use of BN modelling it is being reviewed as it has the potential capability to model the probability of a decision being made based upon varying inputs.

The aim is to factor in a limited number of cultural attributes into the role profiles for the stakeholders within the FPD of a set scenario and then model that scenario for a number of differing environments and situations. The quantification of the attributes will be done through a number of methods including using current cultural knowledge within the field such as [17]; Fig. 11 'illustrates the balance of values and practices for various "levels" of culture' [17].
Further work is to include iterations of expert meetings to establish figures that are realistic for the research and time frame.

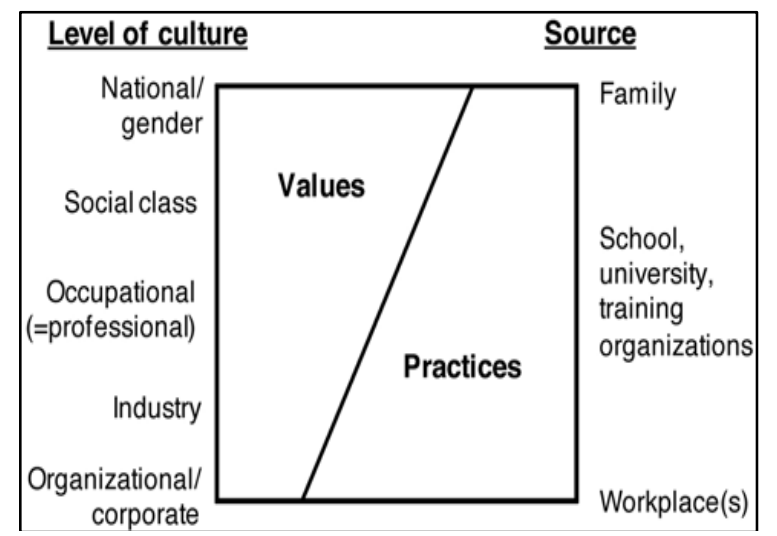

Fig. 11. Hofstede's balance of values and practices [17].

\section{CONCLUSION}

Understanding the customer and the environment within which a system or SoS operates is a major factor in producing effective and successful systems/SoS that can deliver the required capabilities. There has been a large amount of work within this area but the majority of this work focuses solely on specific customers and within isolated operational spaces. This research intends to provide a better understanding of the whole picture via a holistic view of operations and integrating current knowledge and models with a better understanding of influencing factors.

The outcome will be a decision aiding tool within the decision making process. The key challenging areas within this research are the quantification of cultural aspects and modelling their influence upon a decision.

\section{REFERENCES}

[1] ISO/IEC, 15288:2008(en), section 4.31.

[2] M. E. Jamshidi, "System of systems engineering - innovations for the 21st century," J. Wiley \& Sons., 2009.

[3] M. Maier., "Architecting principles for systems of systems," vol. 1, no. 4, 1998.

[4] R. Kalawsky. (2015). Agile wing integration. [Online]. Available: http://www.lboro.ac.uk/research/avrrc/about/news/virtualengineering-for-future-aircraft.html

[5] MoD. (2015). MoD Architecture Framework. [Online]. Available: https://www.gov.uk/mod-architecture-framework.

[6] DoD. (2015). DoD architecture framework. [Online]. Available: http://dodcio.defense.gov/Portals/0/Documents/DODAF/DoDAF_v202_web.pdf

[7] B. P. O. and S. I. Josko, "DANSE - designing for adaptability and evolution in system of systems engineering," 2012.

[8] Compass. (2015). [Online]. Available: http://www.compassresearch.eu/.

[9] V. Senni. (2016). DANSE - designing for adaptability and evolution in systems of systems engineering. [Online]. Available: http://www.cpsos.eu/wpcontent/uploads/2015/07/DANSE_Designing-for-adaptability-andevolution.pdf

[10] D. D. Han SY, "Development interdependency modeling for systemof-systems (sos) using bayesian networks: SOS management strategy planning," Procedia Computer Science, vol. 16, pp. 698-707, 2013.

[11] C. Kadushin, "Chapter 2. some basic network concepts and propositions," In Introduction to Social Network Theory, 2004.

[12] C. S. M. S. Katie Callan, "A case study example of the role matrix technique," International Journal of Project Management, no. 624, pp. 506-515, August 2006. 
[13] I. S. Organisation, "ISO 9000 introduction and support package: Guidance on the concept and use of the process approach for management systems ISO/TC 2008:176/SC," ISO, 2008.

[14] J. D, "The stakeholder management framework for teams, programs and portfolios," Scaled Agile Inc, 2012.

[15] S. J. J. M.-T. J. H. a. E. Y. B. Johansson, "An introduction to systems modelling and simulations with colored ptrei nets," In Winter Simulation Conference: IEEE, 2010.

[16] I. project, "General characterisation of airport processes and its interaction," Seventh Framework Programme.

[17] H. E. S. C. Hodgson A, "Toward an understanding of culture and the performance of teams in complex systems," IEEE Systems Journal, vol. 7, no. 4, pp. 606-615, Dec. 2013.

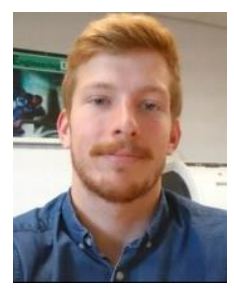

Guy J. Schmidt was born in Pietermaritzburg, South Africa on June 3, 1991. He got the master degree in systems engineering from Loughborough University, United Kingdom in 2014. He got the diploma of Industrial Studies - obtained through an industrial placement year within the degree studying for a $\mathrm{Ph} . \mathrm{D}$ in the area of system of systems engineering focused on the air transport system (ATS).

The placement year was spent with Lockheed Martin UK working on the merlin capability sustainment program; he worked as a systems test engineer during the placement working on the tactical management computer currently studying for a $\mathrm{Ph} . \mathrm{D}$ within the Wolfson school of Mechanical, Electrical and Manufacturing Engineering; current research interests are decision modelling and cultural implications upon this within the ATS. Past research interests include process modelling and disruptive innovations.

Mr. Schmidt, postgraduate systems program representative on the postgraduate committee within Loughborough University. 